\title{
Absorption and Polarization Phenomena in Cross-Linked Polyethylene Films
}

\author{
Margarita E. Borisova ${ }^{1 *}$, Julia K. Osina ${ }^{2}$ \\ ${ }^{1,2}$ Institute of Energy and Transport Systems, Peter the Great St. Petersburg Polytechnic University,
} Russian Federation

\begin{abstract}
The charging and discharging currents of the cable polyethylene insulation before and after thermal aging were experimentally studied and analyzed. Analysis was carried out on the basis of equivalent Maxwell and Voigt circuits. Thermally stimulated depolarization current in open and shorted circuit and the kinetics of charge accumulation were studied. The nature of the charge accumulated in film by applying a constant electric field and the charge storage mechanism were determined.
\end{abstract}

Keywords - Absorption current, cross-linked polyethylene, discharge voltage, equivalent circuit, homocharge, polarization, return voltage, thermal aging.

\section{INTRODUCTION}

Polymer composite materials based on polyethylene are widely used in electrical insulation, capacitor and cable technology where the dielectric ability to accumulate and maintain a charge leads to negative phenomena. The accumulation of volume charge in the operation of a cable at a constant voltage leads to a distortion of the electric field, thereby reducing the dielectric strength and lifetime of the insulation [1]. Therefore, it is profitable to replace cables with paper-impregnated insulation by cables with cross-linked polyethylene insulation, which have a number of advantages. The study of the absorption properties of the cable insulation can provide information about the strength of the insulation affected by aging and the dielectric's ability to store charge. Thus, the study of the absorption characteristics of electrical insulation is relevant.

As is well known [2]-[4], absorption characteristics include charge current $I^{\mathrm{ch}}(t)$ and discharge current $I^{\mathrm{dis}}(t)$, self-discharge voltage $U^{\mathrm{s}}(t)$ and restored voltage $U^{\mathrm{r}}(t)$. On the basis of experimental curves $\underline{I}^{\mathrm{ch}}(t), I^{\mathrm{dis}}(t), U^{\mathrm{s}}(t), U^{\mathrm{r}}(t)$, a calculation of the dependence of frequency on the complex impedance $\dot{Z}(\omega)$ and the complex dielectric permittivity $\varepsilon(\omega)$ over a low frequency range $\left(10^{-5} \mathrm{~s}^{-1}\right.$ to $\left.1 \mathrm{~s}^{-1}\right)$ was made.

\section{EXPERIMENTAL DATA}

The charging and discharging currents of cross-linked polyethylene films were investigated experimentally. The films of peroxide cross-linked PE and silane cross-linked PE were cut from cable insulation. Absorption phenomena in peroxide cross-linked PE (grade composition HFDK 4201 SC, manufactured by "Dow Wire and Cable") and silane crosslinked PE (the composition of the brand LE 4421 (95\%) with a catalyst LE 4431 (5 \%) manufactured by "Borealis") were studied [5].

The thickness $(h)$ of the films was $45 \mu \mathrm{m}$. Aluminum electrodes with a diameter of $20 \mathrm{~mm}$ were used.

The electric field strength was $10^{6} \mathrm{~V} / \mathrm{m}$. The film samples were preliminarily heated up to $90{ }^{\circ} \mathrm{C}$ over one hour.

The time-dependences of charging current $I^{\text {ch }}(t)$ have been examined over the $60{ }^{\circ} \mathrm{C}$ to $90{ }^{\circ} \mathrm{C}$ temperature range. For every sample, measurements were carried out at temperatures from $60{ }^{\circ} \mathrm{C}$ to $90{ }^{\circ} \mathrm{C}$. The currents were measured over $2000 \mathrm{~s}$ to reach stationary values.

In the present study, the influence of thermal aging was investigated for different types of cross-linked polyethylene cable insulation. The thermal aging of the cross-linked PE specimens was performed at $T=90^{\circ} \mathrm{C}$ over $400 \mathrm{~h}$.

The dependences of charging current $I^{\text {ch }}=f(t)$ and discharging current $I^{\mathrm{dis}}=f(t)$ for initial and aged peroxide crosslinked PE (PXLPE) and silane cross-linked PE (SXLPE) films are shown in Fig. 1. The figure shows that the values of absorption current and accumulated charge increased with temperature rise.

After thermal aging, the absorption current and the charge accumulated in the dielectric decrease in PXLPE films as shown in [6] and increase in SXLPE films by the factor of ten. The changes in the absorption current point to changes in the dielectric properties after the thermal aging. It can be seen that the magnitude of the charging current has increased after the aging.

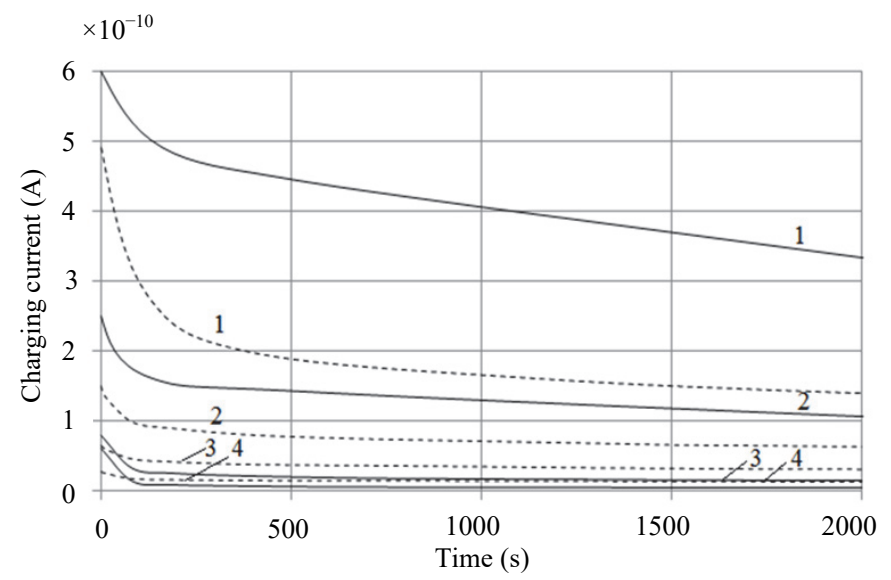

Fig. 1. Charging current as a function of time for initial SXLPE (dotted line) and PXLPE (continuous line) films at different temperatures: $1-90{ }^{\circ} \mathrm{C}$; $2-80{ }^{\circ} \mathrm{C} ; 3-70{ }^{\circ} \mathrm{C}, 4-60{ }^{\circ} \mathrm{C}$.

\footnotetext{
${ }^{*}$ Corresponding author.

E-mail address: vladimirl.borisov@gmail.com
} 
In all the cases, the relation $I^{\mathrm{ch}}(t)-I^{\mathrm{hr}}=-I^{\mathrm{dis}}(t)$ was fulfilled, where $I^{\text {thr }}$ is stationary conductivity current. A comparison between the absorption current of the initial and aged films at $60{ }^{\circ} \mathrm{C}$ to $90{ }^{\circ} \mathrm{C}$ can be seen in figures 1 and 2 .

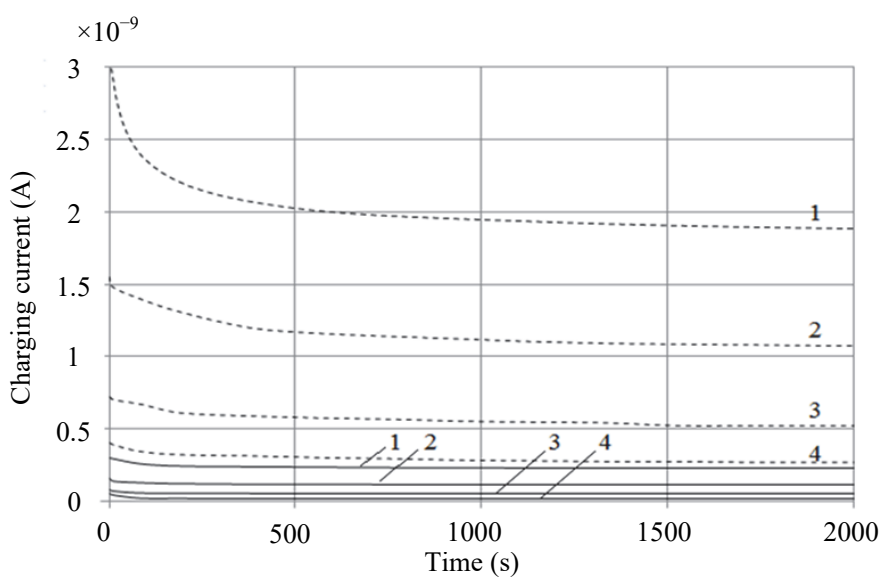

Fig. 2. Charging current as a function of time for aging SXLPE (dotted line) and PXLPE (continuous line) films at different temperatures: $1-90{ }^{\circ} \mathrm{C}$ $2-80{ }^{\circ} \mathrm{C} ; 3-70{ }^{\circ} \mathrm{C}, 4-60{ }^{\circ} \mathrm{C}$.

\section{THEORETICAL ANALYSIS}

The experimentally measured absorption currents were analyzed on the basis of two physical models - Maxwell and Voigt models - and their corresponding equivalent circuits [7], [8].
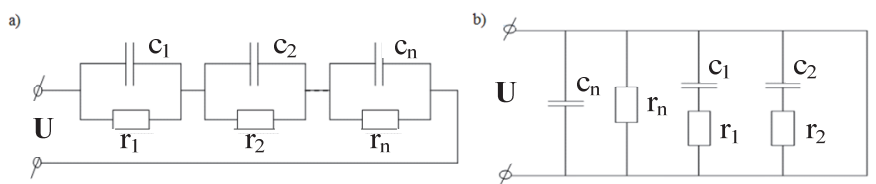

Fig. 3. Maxwell equivalent circuit (a) and Voigt equivalent circuit (b).

The initial differential expression for the Voigt circuit [7], [8] has the form

$$
\begin{gathered}
I^{\mathrm{V}}=\frac{\mathrm{d} q_{n}}{d t}+\frac{q_{n}}{\vartheta_{n}}+\sum_{i=1}^{n-1} \frac{\mathrm{d} q_{i}}{\mathrm{~d} t}, \\
U^{\mathrm{V}}=\frac{q_{n}}{c_{n}}=\frac{q_{i}}{c_{i}}+r_{i} \frac{\mathrm{d} q_{i}}{\mathrm{~d} t},
\end{gathered}
$$

where $q_{n}, q_{i}$ are the charges on capacitances $c_{n}, c_{i} ; \vartheta_{n}=c_{n} r_{n}$ and $\vartheta_{i}=c_{i} r_{i}$ - the relaxation times, $i=1,2, \ldots,(n-1)$.

Initial conditions for charging:

$$
q_{i}(0)=0 ; q_{n}(0)=c_{n} U_{\mathrm{p}} .
$$

Using the initial conditions (3), we get the expressions for the current of electrical insulation in the case of the relaxation polarization model, or Voigt equivalent circuit.

$$
\frac{I^{\mathrm{ch}}(t)-I^{\mathrm{thr}}}{I^{\mathrm{thr}}}=-\frac{I^{\mathrm{dis}}(t)}{I^{\mathrm{thr}}}=\sum_{i=1}^{n-1} \frac{r_{n}}{r_{i}} \exp \left(-\frac{t}{\vartheta_{i}}\right),
$$

where $I^{\text {thr }}$ stands for the through current, $I^{\text {thr }}=U_{\mathrm{p}} / r_{n}$.

Initial conditions in the analysis of voltage response and discharge voltage:

$$
\begin{gathered}
q_{n}(0)=c_{n} U_{\mathrm{p}} ; \quad q_{i}(0)=c_{i} U_{\mathrm{p}} ; \\
\frac{U^{\mathrm{s}}(t)}{U_{\mathrm{p}}}=\sum_{i=1}^{n} \frac{\prod_{k=1}^{n-1}\left(\tau_{i}-\vartheta_{k}\right)}{\prod_{k \neq i}^{n}\left(\tau_{i}-\tau_{k}\right)} \exp \left(-\frac{t}{\vartheta_{i}}\right) \\
\frac{U^{\mathrm{r}}(t)}{U_{\mathrm{p}}}=\sum_{i=1}^{n} \frac{\prod_{k=1}^{n}\left(\tau_{i}-\vartheta_{k}\right)}{\tau_{i} \prod_{k \neq i}^{n}\left(\tau_{i}-\tau_{k}\right)} \exp \left(-\frac{t}{\tau_{i}}\right)
\end{gathered}
$$

To calculate the dependences $U^{\mathrm{s}}(t)$ and $U^{\mathrm{r}}(t)$, it is necessary to know the time constants $\tau_{i}$ that can be calculated from the characteristic equation.

For the model of a multilayer dielectric and the corresponding Maxwell equivalent circuit, the system of differential equations may be written as follows:

$$
\begin{aligned}
& I^{\mathrm{M}}=\frac{Q_{i}}{\tau_{i}}+\frac{\mathrm{d} Q_{i}}{\mathrm{~d} t}, \\
& U^{\mathrm{M}}=\sum_{i=1}^{n} \frac{Q_{i}}{C_{i}},
\end{aligned}
$$

where $Q_{i}$ is the charge on capacitance $C_{i}$ and $\tau_{i}=R_{\mathrm{i}} C_{\mathrm{i}}$ - the relaxation time, $i=1,2, \ldots, n$.

The initial conditions for the calculation of $U^{\mathrm{s}}(t), U^{\mathrm{r}}(t), I^{\mathrm{ch}}(t)$, $I^{\text {dis }}(t)$ after complete charging of the dielectric are of the following form:

$$
I^{\mathrm{M}}=0, Q_{i}^{c}(0)=U_{\mathrm{p}} \frac{\tau_{i}}{R_{\Sigma}}, Q^{s}{ }_{i}(0)=\frac{U_{\mathrm{p}}}{R_{\Sigma}}\left(\tau_{i}-\tau_{\Sigma}\right) .
$$

Using the initial condition (9) and Laplace transformation, we can obtain the final solutions for charging and discharging currents, for decay voltage and return voltage.

$$
\begin{gathered}
\frac{I^{\mathrm{ch}}(t)-I^{\mathrm{thr}}}{I^{\mathrm{thr}}}=-\frac{I^{\mathrm{dis}}(t)}{I^{\mathrm{thr}}}=-\sum_{i=1}^{n-1} \frac{\prod_{k=1}^{n}\left(\vartheta_{i}-\tau_{k}\right)}{\vartheta_{i}^{2} \prod_{k \neq i}^{n-1}\left(\vartheta_{i}-\vartheta_{k}\right)} \exp \left(-\frac{t}{\vartheta_{i}}\right) \\
\frac{U^{\mathrm{s}}(t)}{U_{\mathrm{p}}}=\sum_{i=1}^{n} \frac{R_{i}}{R_{\sum}} \exp \left(\frac{t}{\tau_{i}}\right) \\
\frac{U^{\mathrm{r}}(t)}{U_{\mathrm{p}}}=\sum_{i=1}^{n} \frac{R_{i}}{R_{\Sigma}}\left(1-\frac{\vartheta_{n}}{\tau_{i}}\right) \exp \left(\frac{t}{\tau_{i}}\right)
\end{gathered}
$$

where $U_{\mathrm{p}}$ is the polarization voltage, $R_{\Sigma}=\sum R_{i}, I^{\mathrm{thr}}=U_{\mathrm{p}} / R_{\Sigma}$ is the through current.

$$
\tau_{i}=R_{i} C_{i} ; \quad \tau_{\Sigma}=R_{\Sigma} C_{\Sigma}
$$




$$
C_{i}=\left[\sum_{i=1}^{n} C_{i}^{-1}\right]^{-1}=C_{n}
$$

From experimental dependence Us(t), which consists of the sum of sum of exponents (11), the parameters of Maxwell equivalent circuit can be determined.In the paper [7], it is shown that the absorption characteristics of the Maxwell circuit and the Voigt circuit are identical when the number of element pairs $\mathrm{n}$ is equaled and the following conditions are fulfilled:

$$
\begin{aligned}
& \tau_{i}^{\mathrm{M}}=\tau_{i}^{\mathrm{V}}=\tau_{i}, \vartheta_{i}^{\mathrm{M}}=\vartheta_{i}^{\mathrm{V}}=\vartheta_{i}, \tau_{\sum}=\vartheta_{n}, \\
& C_{\Sigma}=c_{n}, R_{\Sigma}=\sum R_{i}=r_{n}, \\
& \frac{r_{n}}{r_{i}}=\frac{R_{i}}{R_{\Sigma}} .
\end{aligned}
$$

The frequency characteristics of the Maxwell equivalent circuit are easy to obtain by using a symbolic method:

$$
\dot{Z}(\omega)=\sum_{i=1}^{n} \frac{R_{i}}{1+i \omega \tau_{i}}=\sum_{i=1}^{n} \frac{R_{i}}{1+\omega^{2} \tau_{i}^{2}}-i \sum_{i=1}^{n} \frac{R_{i} \omega \tau_{i}}{1+\omega^{2} \tau_{i}^{2}}=Z^{\prime}-i Z^{\prime \prime},
$$

where $Z$ and $Z^{\prime \prime}$ are the active and reactive components of the complex impedance. The complex conductivity is calculated from the following formula:

$$
\dot{Y}_{\mathrm{M}}(\omega)=\frac{\prod_{i=1}^{n}\left(1+i \omega \tau_{i}\right)}{\sum_{i=1}^{n} R_{i} \prod_{k \neq i}^{n}\left(1+i \omega \tau_{k}\right)}=i \omega C_{0}\left(\varepsilon^{\prime}-i \varepsilon^{\prime \prime}\right)
$$

As a result, we obtain expressions for the frequency characteristics of the equivalent Maxwell circuit corresponding to a multi-layer dielectric.

\section{ANALYSIS OF EXPERIMENTAL DATA}

The experimentally measured absorption currents were analyzed by means of formula (4) (Voigt circuit). The dependences of charging current are described by the sum of two exponents with relaxation times $\vartheta_{1}$ and $\vartheta_{2}$ :

$$
I=I_{01} \mathrm{e}^{-t / \vartheta_{1}}+I_{02} \mathrm{e}^{-1 / \vartheta_{2}}
$$

This expression corresponds to the six-element Voigt circuit, whose parameters are determined by expressions (18):

$$
r_{n}=\frac{U_{\mathrm{p}}}{I^{t h \mathrm{~h}}} ; c_{n}=\frac{\varepsilon_{0} \varepsilon S}{h} ; \vartheta_{n}=c_{n} r_{n} ; c_{i}=\frac{\varepsilon_{0} \varepsilon_{s i} S}{h} ; r_{i}=\frac{\vartheta_{i}}{c_{i}}=\frac{h \vartheta_{i}}{\varepsilon_{0} \varepsilon_{s i} S}
$$

$\varepsilon_{s i}$ - permittivity of each layer; $\gamma_{i}$ - conductivity; $h_{i}$ - layer thickness, $S$ - electrode area; $i=1,2, \ldots,(n-1)$. Highfrequency capacitance $c_{n}$ related to quickly established polarization was measured on an LCR meter E7-8 at $1000 \mathrm{~Hz}$. For PXLPE films $c_{n}=8.0 \times 10^{-11} \mathrm{~F}$ and for SXLPE films $c_{n}=$ $9.1 \times 10^{-11} \mathrm{~F}$.

Defining the parameters of the Voigt equivalent circuit and considering the adequacy of the two equivalent circuits [7], [8],

\begin{tabular}{|c|c|c|c|c|c|c|}
\hline$\vartheta_{1}, s$ & $\vartheta_{2}, \mathrm{~s}$ & $\begin{array}{c}r_{1}, \times 10^{12} \\
\Omega\end{array}$ & $\begin{array}{c}r_{2}, \times 10^{12} \\
\Omega\end{array}$ & $\begin{array}{c}c_{1}, \\
\times 10^{-11} \\
\mathbf{F} \\
\end{array}$ & $\begin{array}{c}c_{2}, \\
\times 10^{-11} \\
\mathbf{F} \\
\end{array}$ & $T,{ }^{\circ} \mathbf{C}$ \\
\hline \multicolumn{7}{|c|}{ PXLPE } \\
\hline 2455 & 300 & 150 & 5,5 & 1,6 & 5,4 & 60 \\
\hline 2350 & 280 & 66 & 4,9 & 3,5 & 5,7 & 70 \\
\hline 2050 & 123 & 5,8 & 3,4 & 34 & 3,6 & 80 \\
\hline 1600 & 63 & 1,4 & 3,9 & 110 & 1,6 & 90 \\
\hline \multicolumn{7}{|c|}{ SXLPE } \\
\hline 2912 & 393 & 150 & 22 & 1,9 & 1,7 & 60 \\
\hline 2420 & 200 & 27 & 12 & 8,8 & 1,6 & 70 \\
\hline 1916 & 190 & 13 & 4,5 & 15 & 4,2 & 80 \\
\hline 1555 & 156 & 3,1 & 1,2 & 49 & 0,1 & 90 \\
\hline
\end{tabular}
we may transit to the Maxwell equivalent circuit.

The parameters of the Voigt equivalent circuit and the Maxwell equivalent circuit are presented in Table I and Table II.

\begin{tabular}{|c|c|c|c|c|c|}
\hline $\begin{aligned} & R_{1}, \\
\times & 10^{11} \Omega\end{aligned}$ & $\begin{aligned} & R_{2}, \\
& \times 10^{11} \Omega\end{aligned}$ & $\begin{aligned} & R_{3}, \\
& \times 10^{11} \Omega\end{aligned}$ & $\begin{array}{c}C_{1}, \\
\times 10^{-10} \\
F\end{array}$ & $\begin{array}{c}C_{2} \\
\times 10^{-10} \\
F\end{array}$ & $\begin{array}{c}C_{3}, \\
\times 10^{-10} \mathrm{~F}\end{array}$ \\
\hline & \multicolumn{3}{|c|}{ PXLPE at $80^{\circ} \mathrm{C}$} & & \\
\hline \multirow[t]{2}{*}{5.0} & 10.0 & 12.2 & 1.33 & 31.2 & 2.1 \\
\hline & \multicolumn{3}{|c|}{ PXLPE at $90^{\circ} \mathrm{C}$} & & \\
\hline \multirow[t]{2}{*}{3.3} & 4.1 & 2.3 & 1.0 & 66.7 & 3.7 \\
\hline & \multicolumn{3}{|c|}{ SXLPE at $80^{\circ} \mathrm{C}$} & & \\
\hline \multirow[t]{2}{*}{6.3} & 19.8 & 23.8 & 1.7 & 14.5 & 2.2 \\
\hline & \multicolumn{3}{|c|}{ SXLPE at $90^{\circ} \mathrm{C}$} & & \\
\hline 4.8 & 9.0 & 7.6 & 1.1 & 28.0 & 4.4 \\
\hline
\end{tabular}

TABLE I

The Parameters of THE Voigt EquiValent Circuit FOR XLPE FILMS

TABLE II

The Parameters of The MaXwell EQuivalent Circuit FOR XLPE FILMS

The dependences $U^{\mathrm{s}}(t)$ and $U^{\mathrm{r}}(t)$ for cross-linked polyethylene films were calculated using expression (11) and the parameters of the Maxwell circuit (Table II). Figure 4 shows the dependence $U^{s}(t)$ for the SXLPE film. As the temperature increases, there is a reduction in the value of $U^{\mathrm{s}}$ and maximum $U^{\mathrm{r}}$ is moved to the region of the small values of time. 


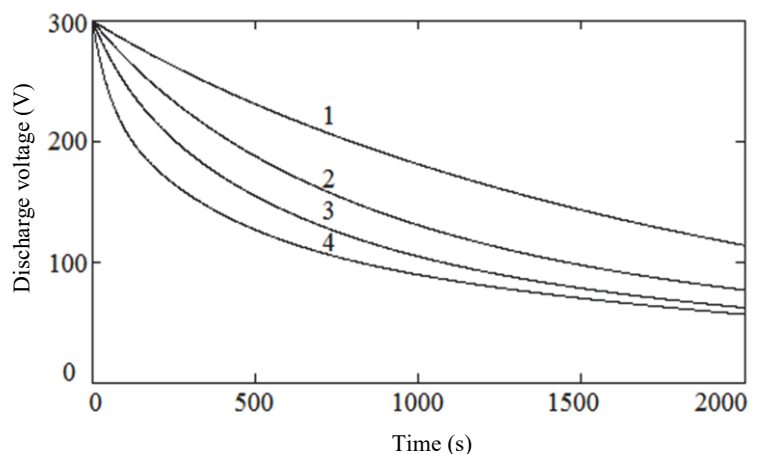

Fig. 4. The dependence $U^{\mathrm{s}}=f(t)$ for the SXLPE film at different temperatures: $1-60{ }^{\circ} \mathrm{C} ; 2-70{ }^{\circ} \mathrm{C} ; 3-80{ }^{\circ} \mathrm{C}, 4-90{ }^{\circ} \mathrm{C}$.

The dependences of the active and reactive components of the complex impedance were calculated with the help of expression (15) and for SXLPE film these are shown in Figure 5.

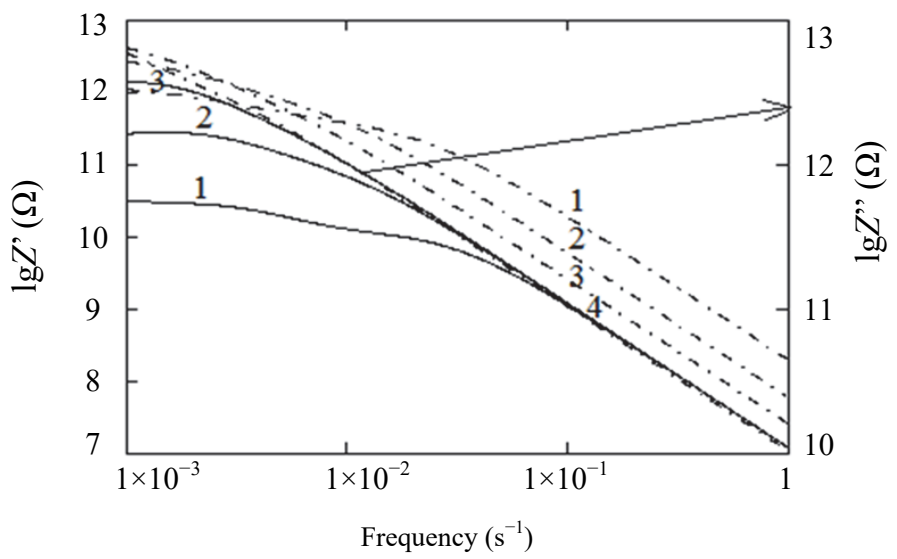

Fig. 5. Dependences $\lg Z=f(\omega)$ and $\lg Z^{\prime \prime}=f(\omega)$ of the initial SXLPE samples at different temperatures: $1-90{ }^{\circ} \mathrm{C} ; 2-80{ }^{\circ} \mathrm{C} ; 3-70{ }^{\circ} \mathrm{C} ; 4-60{ }^{\circ} \mathrm{C}$.

It is found that in the initial SXLPE and PXLPE samples the reactive components of the complex impedance at $90{ }^{\circ} \mathrm{C}$ and $\omega=10^{-3} \mathrm{~s}^{-1}$ were almost equal $\left(Z^{\prime \prime}=2.0 \times 10^{11} \Omega\right.$ and $5.0 \times 10^{11} \Omega$ ) but the value of $Z^{\prime}$ was greater for SXLPE than for PXLPE. As a result of the thermal aging, the values of $Z^{\prime}$ and $Z$ " for SXLPE diminished by an order of magnitude but the value of $Z(\omega)$ remained unchanged for PXLPE.

The kinetics of charging of the SXLPE films was studied in a constant electric field with a strength of $E=6 \times 10^{6} \mathrm{~V} / \mathrm{m}$. The sample was kept in a heating cell at $T=70^{\circ} \mathrm{C}$. The charge $\sigma$ accumulated in the film under the action of the electric field was measured after every $10 \mathrm{~min}$. The sign and value of the charge were measured by the method of electrostatic induction. It was established that homocharge is accumulated in film and the dependence $\sigma(t)$ is described by a smoothly increasing curve.

To study the processes occurring in the dielectric, the method of thermally stimulated depolarization current (TSD current) was used. Before measuring the TSD current, the SXLPE samples were pre-polarized to $400 \mathrm{~V} \pm 50 \mathrm{~V}$ in a corona discharge at corona electrode negative polarity. The TSD current was measured in open circuit [5]. Thus, a 20micrometer-thick polytetrafluoroethylene film was placed between the $\mathrm{Al}$ electrode and the charged sample. The tests were conducted on a series of identical samples of different types of chemical cross-linked PE.

It was established that during the transition from the close contact (in a short circuit) to the loose contact (in an open circuit), sign inversion of $I^{\mathrm{TSD}}$ takes place, thus testifying to the presence of homocharge in the sample [9]. The direction of current at close contact is due to the increased conductivity of the surface layer of the film [10].

\section{CONCLUSION}

The absorption characteristics for the XLPE samples were measured and calculated before and after the thermal aging. Analysis of these dependences was performed on the basis of two six-element Maxwell and Voigt equivalent circuits. The obtained time and frequency characteristics turned out to be completely identical for both circuits.

It was found that in XLPE samples, when placed in a constant electric field of $10^{6} \mathrm{~V} / \mathrm{m}$, homocharge accumulates. The dependence of the charge on time is described by a smoothly rising curve, tending towards a limiting value. It is shown that the accumulation of homocharge occurs due to MaxwellWagner polarization.

The value of the charge stored in the SXLPE film is several times greater than in the case with the PXLPE film. Therefore, with cables operating at a constant voltage it is recommended to use PXLPE film.

\section{REFERENCES}

[1] G. C. Montanari, "The electrical degradation threshold of polyethylene investigated by space charge and conduction current measurements," IEEE Transactions on Dielectrics and Electrical Insulation, vol. 7, no. 3, pp. 309-315, Jun. 2000. https://doi.org/10.1109/94.848905

[2] T. K. Saha, J. H, Yew, and P. Purkait, "Experience with dielectric response measurements on oil-paper insulated cables," in XIIIth International Symposium on High Voltage Engineering, Netherlands 2003. pp. 1-4.

[3] Nemeth, E. The Fundamentals of Diagnostic Testing of Electrical. Proceedings of the international scientific-practical conference "Electrical Insulation -99”, St. Petersburg. - 1999. pp. 9-12.

[4] J. Lowell, "Absorption and conduction currents in polymers: a unified model," Journal of Physics D: Applied Physics, vol. 23, no. 2, pp. 205210, Feb. 1990. https://doi.org/10.1088/0022-3727/23/2/011

[5] M. E. Borisova and Y. K. Osina, "Analysis of relaxation processes by mathematical modeling," Technical Physics Letters, vol. 41, no. 8, pp. 717-719, Aug. 2015. https://doi.org/10.1134/s1063785015080052

[6] C. Stancu, P. V. Notingher, P. Notingher jr. et al., "Study of thermally and water tree aged polyethylene by infrared spectroscopy, absorption currents and space charge measurements," in Proceedings of 7th International Conference on Insulated Power Cables, France, ParisVersailles, June 24-28, 2007, pp. 758-763.

[7] M. E. Borisova, S. N. Koikov, M. S. Marchenko, and N. V. Severyukhina, "Evaluation of the parameters of dielectric non-homogeneity by analysis of absorption characteristics," Electrical Technology, no. 2, pp. 139-149, 1995.

[8] M. E. Borisova, O. V. Galyukov and P. V. Tsatsynkin, "Low-frequency dependence of dielectric characteristics of polyimide films," in Proceedings of the 5th International conference "Electromechanics, Electrotechnology and electromaterials”, Alushta, 2003, pp. 17-20.

[9] G. M. Sessler Ed., Electrets, Springer-Verlag, Berlin-Heidelberg-New York, 1987. https://doi.org/10.1007/3-540-17335-8

[10] M. E. Borisova, I. J. Gallay and S. N. Koikov, "Low-frequency dispersion of the capacitance film samples of polyethylene terephthalate," Electrical Technology, no. 3, pp. 69-71, 1993. 